\title{
A Radio-Optical Reference Frame VIII. CCD observations from KPNO and CTIO: internal calibration and first results
}

\author{
N. Zacharias ${ }^{1}$, C. de Vegt $^{2}$, L. Winter ${ }^{2}$, K.J. Johnston \\ U.S. Naval Observatory, 3450 Mass. Ave. N.W., Washington D.C. 20392
}

Received __; accepted _

AJ manuscript, revised version for submission, 03 August 95

\footnotetext{
${ }^{1}$ with Universities Space Research Association (USRA), Division of Astronomy and Space Physics, Washington D.C.

${ }^{2}$ Hamburger Sternwarte, University of Hamburg, F.R. of Germany
} 


\begin{abstract}
In this pilot investigation, precise optical positions in the FK5 system are presented for a set of 16 compact extragalactic radio sources, which will be part of the future radio-optical reference frame. The $0.9 \mathrm{~m} \mathrm{KPNO}$ and CTIO telescopes equipped with $2 \mathrm{~K}$ CCD's have been used for this project. The astrometric properties of these instruments are investigated in detail. New techniques of using wide field CCD observations for astrometry in general are developed. An internal precision of 5 to 31 mas in position per single exposure is found, depending on the brightness of the object. The tie to the primary optical reference system is established by photographic astrometry using dedicated astrographs on both hemispheres. An accuracy of $\approx 30$ mas per source is estimated for the multi-step reduction procedure when based on the future Hipparcos catalog, while the FK5-based positions suffer from system errors of 100 to 200 mas as compared to the radio positions. This work provides a contribution to the international effort to link the Hipparcos instrumental coordinate system to the quasi-inertial VLBI radio reference frame. Precise radio and optical astrometry of a large sample of compact extragalactic sources will also contribute to the astrophysics of these objects by comparing the respective centers of emission at the optical and radio wavelengths.
\end{abstract}

Subject headings: astrometry: reference frame, CCD observation and reduction techniques - Hipparcos: extragalactic reference frame link — QSO, BL Lac: optical positions 


\section{Introduction}

This paper is part of a series of papers describing the construction and maintenance of a quasi-inertial reference frame in both the radio and optical domain.

Extensive work on the radio reference frame has been accomplished in the last 5 years and published in a series of papers I-VII (see Johnston et al. 1995 for further details). A rigorous global new reduction of all then applicable Mark III VLBI radio observations has been used to construct a radio reference frame of 560 sources from first principles (Johnston et al. 1995). Based on these results a list of defining and candidate sources has been provided to the IAU Working Group on Reference Frames (IAU, 1995). While a dense

radio frame with an accuracy level of 1 milliarcsecond (mas) for most of the source positions is now in place, optical observations on a 30-50 mas level are available for only a fraction of these sources.

Previous results already have shown the deficiencies of the currently used optical FK5/J2000 reference frame, with deviations from a uniform inertial reference system as large as $\approx 200$ mas at the current epoch. The Hipparcos astrometry satellite mission will soon provide a new optical system on the 2 mas level at the Hipparcos mean epoch $(\approx 1991.5)$, and 2 mas/year in proper motion error, but this instrumental system must be linked to the radio system to become quasi-inertial. Most of the primary optical reference objects (stars) are bright, both in the FK5 (3 to 6 mag) and the Hipparcos (5 to 9 mag) catalogs, while the optical counterparts of the extragalactic sources are optically faint (the majority in the range 17 to $21 \mathrm{mag})$.

Due to the relatively low quantum efficiency of photographic astrometry, which requires long exposure times on large telescopes in good seeing, progress in the optical observations has been slow. The use of CCD detectors has dramatically improved this situation, because they allow smaller telescopes which have greater availability and more objects can be 
observed due to much shorter exposure times. Not until recently has the fieldsize of CCD's became large enough to contain a sufficient number of reference stars for precise astrometry.

This paper outlines the reduction procedure in detail and gives results for a representative subset of the observed sources. It is a pilot investigation to assess the astrometric properties of these telescopes and the capabilities of this technique. The first successful attempt to use CCD's for this project has been made earlier (de Vegt et al. 1987), although it was severely limited by the lack of reference stars in a tiny 2 ' by 3 ' field of view.

In Section 2 we discuss the telescopes, CCD's and observations and in Section 3 the reference star data. Section 4 describes the reduction procedure, while results are presented in Section 5. An accuracy estimate of the procedure and comparison with other investigations is made in Section 6.

\section{OBSERVATIONS}

\subsection{The Telescopes}

The $0.9 \mathrm{~m}$ (36 in) Kitt Peak National Observatory (KPNO) telescope is a RitcheyChrétien system with an additional 2-element field corrector about $250 \mathrm{~mm}$ before the focal plane. This gives a large $(\approx 1 \mathrm{deg})$ flat field of view, which also gives the offaxis guide scope good image quality. Frequent focus measurements and the use of the correlation between focus setting and telescope temperature ensured optimal image quality for all object frames. The image quality at the KPNO instrument was found to be uniformly good over the entire field of the CCD.

The $0.9 \mathrm{~m}$ (36 in) Cerro Tololo Inter-American Observatory (CTIO) telescope is a cassegrain system without a field corrector. At the edge of the CCD frame optical 
aberrations are visible and it is difficult to achieve a well focused CCD frame with round images over most of the chip area due to instabilities in the mirror-supporting structure. We also had occasional guiding problems. On the positive side the longer focal length of the CTIO $0.9 \mathrm{~m}$ with its better sampling, makes it more suitable for structure analysis of the objects than the KPNO $0.9 \mathrm{~m}$. Getting enough reference stars for the astrometric link was found to be the bottleneck with the CTIO $0.9 \mathrm{~m}$.

Properties of both telescopes and the CCD's used are summarized in Table 1.

\subsection{The Data Acquisition System}

Both telescopes use the same type of Tektronix 2K CCD chip, with square pixels of size $24 \mu \mathrm{m}$ and a filling factor of $100 \%$, with a different camera controller. For good astrometric results, a large $\mathrm{S} / \mathrm{N}$ ratio is required, whereas an optimized digitization at the background level is of minor importance. In order to cover the large magnitude range between the reference stars and the extragalactic sources, a large gain of 8.2 was chosen for the KPNO instrument to utilize the full dynamic range, including the full well capacity of the chip. For the CTIO camera, a gain of 3.3 was sufficient because of the larger digitization range (16 bit) available with that camera controller.

The readout time for the KPNO instrument was well over 2 minutes. The new ARCON controller at CTIO allowed a faster readout of the full frame in about 70 seconds with two readout amplifiers.

All frames have been taken in a red spectral bandpass. A Gunn $r$ filter was used for the long exposure frames to record the extragalactic objects. For each object, additional short exposure frames have been taken in order to get unsaturated images of the reference stars (12 to $14 \mathrm{mag}$ ). With the KPNO $0.9 \mathrm{~m}$, most of these short exposure frames have 
been taken with a narrow $(\mathrm{FWHM}=12 \mathrm{~nm})$ filter centered near $H \alpha$. The better sampling of the CTIO instrument allowed the use of the same Gunn $r$ filter for the short exposure frames because the flux of the secondary reference stars was spread out over more pixels, thus avoiding saturation.

The IRAF software system was used for the data acquisition at both sites.

\subsection{Observation Procedure}

A summary of the observing runs is presented in Table 2. Between April and October 1994 the dome seeing was improved at the $0.9 \mathrm{~m} \mathrm{KPNO}$ telescope. In the first observing run at each site many calibration and test frames were obtained in order to evaluate the astrometric quality and possible systematic errors of the instrumentation, as well as to determine the best observing strategy.

The second runs at each telescope were pure production runs, taking at least 2 long and 2 short exposure frames per field. Depending on the brightness of the sources, an exposure time of 200 to 900 seconds was used for the deep frames and 40 to 120 seconds for the others. At the KPNO telescope a typical deep frame covers an astrometrically useable range of 14 to $20^{m}$, while the short exposures cover 10.5 to $16.5^{m}$. At CTIO the corresponding ranges are 13 to $20^{m}$ and 11 to $18^{m}$. All frames were taken within 1 hour of the meridian and at least $30 \mathrm{deg}$ away from the Moon.

In fields of high galactic latitude the density of the secondary reference stars are usually not sufficient for a good astrometric reduction. In those cases a mosaic of short exposures ( $2 \times 2$ frames), centered on the object and shifted by 500" in $x$ and $y$ were obtained at KPNO. The overlap of $41 \%$ in area allows a rigid tie to the central frames and the area covered for potential reference stars was increased by a factor of 2.5. Because of the much 
smaller useable field size of the CTIO $0.9 \mathrm{~m}$, a similar $2 \times 2$ mosaic with shifts of 240 " in $\mathrm{x}$ and y were obtained for all fields at that telescope. This is an overlap of $50 \%$ by area.

A few additional calibration fields were taken with at least a $3 \times 3$ set of frames of 2-3 minutes exposure time. On some nights, additional sets of short exposure (10, 20, 40 seconds) frames were obtained in order to investigate the limits set by atmospheric turbulence on astrometric accuracy. Results will be published elsewhere (Zacharias 1996).

It was necessary to have two observers present, in order to obtain online quality control. On each deep frame, the object was identified and radial profile and contour maps were generated. A few objects were found to be optical doubles on the 2 to 7 arcsecond level, most likely due to foreground stars. Depending on the seeing, these objects were skipped or the exposure time was adjusted, if required. All frames were checked for focus and overall image quality. The short exposures in addition were checked for saturation of the reference star images and the exposure time was adjusted accordingly.

\section{Reference Stars}

The currently used primary reference system is the IRS (International Reference Stars), (Corbin \& Urban 1990, Corbin \& Warren 1991). The IRS gives positions and proper motions in the FK5/J2000 system for approximately 36,000 stars in the magnitude range of $V \approx 6$ to 9 , nearly uniformly distributed on the sky.

Nearly all radio source fields from our candidate list (Johnston et al. 1995) already were observed with modern high precision astrographs. The northern hemisphere plates were taken with the $23 \mathrm{~cm}$ Hamburg Zone Astrograph (ZA) (de Vegt 1978) and the southern hemisphere plates were obtained with the yellow lens (BY) of the U.S. Naval Observatory 8 in Twin Astrograph (Routly 1983) from the Black Birch Astrometric 
Observatory (BBAO) in New Zealand. The field sizes of these instruments are $6 \mathrm{deg} \times 6 \mathrm{deg}$ (ZA) and $5 \mathrm{deg} \times 5 \mathrm{deg}(\mathrm{BY})$ respectively. Both instruments use $6^{m}$ objective gratings in order to obtain diffraction images of bright stars for high precision position measurements. The range of magnitudes covered is $V \approx 5$ to 14 in 15 -minute exposures on microflat $103 a \mathrm{G}$ emulsion. Both instruments have 2 meter focal length, corresponding to a plate scale of about $100 " / \mathrm{mm}$.

All IRS and Hipparcos Input Catalog (HIC) stars (Turon et al. 1992) have been measured on these plates together with all faint stars to the plate limit in an area of $1 \mathrm{deg} \times 1 \mathrm{deg}$ centered on the radio source position. These stars in the magnitude range of $V \approx 11$ to 14 serve as secondary reference stars for the reduction of the CCD frames.

In addition, the $0.5 \mathrm{~m}$ Lick Carnegie Astrograph (LA) was used to provide a denser net of secondary reference stars in selected fields. The LA has a field size of $\approx 3 \mathrm{deg} \times 3 \mathrm{deg}$ on $240 \mathrm{~mm} \times 240 \mathrm{~mm}$ plates but a limiting magnitude of $V \approx 15.5$ in 30 -minute exposures with a plate scale of $55 " / \mathrm{mm}$. For many fields on the southern hemisphere, plates from the ESO Schmidt telescope are available, which often show measurable images of the radio sources. All Schmidt plates provide at least a set of tertiary reference stars in the magnitude range of $R \approx 14$ to 18 . Measuring of all those plates is in progress at Hamburg Observatory with the HAM-I machine (Winter et al. 1992, Winter 1994, Zacharias et al. 1994). An accuracy of $\approx 0.8 \mu \mathrm{m}$ per coordinate is obtained for the measurement of a single image on a good astrograph plate.

For the present pilot study only a subset of CCD observations were used to obtain positions of the optical counterparts of the extragalactic sources. A publication for all fields is in progress and will be based on the Hipparcos catalog.

Ultimately all CCD frames of the $0.9 \mathrm{~m}$ telescopes depend entirely on the astrograph observations, which provide the high precision secondary reference star positions. The 
Guide Star Catalog (GSC) is not precise enough for this project and results from CCD transit circle instruments are premature at the moment. The future Tycho catalog alone will not be dense enough to provide a good reduction of the current CCD observations.

\section{Reduction Procedure}

\subsection{Calibration of Raw CCD Frames}

The standard IRAF software package (version 2.10, NOAO, Univ. of Arizona, Tucson) was used for the initial reduction steps of the raw CCD data. About 20 bias frames were combined for a master Zero for each night. Most of the KPNO frames were calibrated with flatfields derived from a large number of object frames from a group of nights in which the dust grain pattern on the filter and dewar window remained constant. CCD frames taken with the narrow filter at KPNO and all CTIO frames were calibrated with twilight flats.

\subsection{Pre-processing Statistics}

Statistical information such as noise characteristics of the frame and full width half maximum (FWHM) values of the image profiles were obtained from all calibrated images using standard IRAF commands. An approximate pixel position and count rate of the extragalactic objects were obtained from radial profile fits, as well as relative position offsets for the short exposure frames. This information is read by our reduction programs for an automatic data handling of multiple frames and fields. 


\section{3. $\quad$ Determination of $x, y$ Coordinates}

In order to obtain centroid positions of star images $(x, y$ coordinates) two different software packages were investigated for comparison. First, the IRAF/DAOPHOT routines provided three different sets of $x, y$ coordinates: a) a center of mass position, b) a $1-\mathrm{D}$ gaussian fit to the $x, y$ marginal distribution of the pixel data, and c) a point spread function (PSF) fit after subtraction of a gaussian. Only options a) and b), provided by the routine phot, include an error estimate on the derived $x, y$. Option c) is obtained with allstar (Stetson 1987) which can handle crowded fields by a simultaneous fit of profiles to a group of stars and gives superior photometric results, but is not designed for astrometry. Second, $S A A C$ (Software for Analysing Astrometric CCD's) was used. SAAC was developed at Hamburg Observatory (Winter 1994) with various modifications and adaptions performed by N.Zacharias at USNO and performs 2-D fits on the star profiles with a choice of various models.

Slightly saturated images of bright stars usually are of good astrometric quality as long as there is no bleeding into adjacent pixels. These images were kept, with caution, in the following reduction steps. Both program packages exclude significantly elongated images, mostly galaxies and various defects due to restrictions imposed on various image selection parameters.

Positions of radio sources presented in this paper are based exclusively on the circular gaussian 2-D fit, which proved to be the best option for astrometry. For optical doubles the same fit model was used either with the cut-out or with a fit-up procedure (Schramm 1988) or both. In the cut-out option all pixels containing the companion image are excluded from the profile fit observation equations (interactively selected). In the fit-up procedure

the background level is raised to a higher value up to the maximum count rate from the companion within the used window. Bright companion star images were first fitted by these 
methods exclucing the image of the etragalactic source. The thus obtained image profile was subtracted from the original pixel data and finally the position of the extragalactic source determined. The allstar(DAOPHOT) procedure was used for comparison in some cases.

\subsection{Comparison of $x, y$ Data}

Unique star numbers were assigned to all images of all stars of each field using our multi-step match program, based on position only. Coordinates were corrected for approximate third-order optical distortion, derived from a pilot investigation of a subset of the frames. Then all matches within a large search radius are recorded and the distribution of the coordinate differences of the matches in $\mathrm{x}$ and $\mathrm{y}$ are analyzed for a peak. Only the most likely identified matched images are used for a linear transformation using an unweighted least squares adjustment. Finally the transformed coordinates are compared to the reference coordinates and a position match with small tolerance is performed. Optionally, all images identified as multiple on a few arcseconds level are rejected except for manual 'fine tuning' for some radio source images.

A transformation program was developed for comparing $x, y$ data of all overlapping CCD frames of the same field. Various mapping models are available and the residuals can be analyzed and displayed with already existing software from the photographic plate

reduction package. The transformation program was used for comparing $x, y$ coordinates of the same CCD frame as obtained by different pixel fit algorithms, as well as to estimate field distortions and positional accuracy. Optionally, the obtained transformation parameters were applied to combine the $x, y$ data of all frames of each field into a superframe, from which spherical coordinates could be derived. 


\subsection{Combining $x, y$ Data}

One option to derive positions from the combination of all CCD frames of a field is the traditional way of adjusting each individual CCD frame's $x, y$ data to spherical coordinates $(\alpha, \delta)$ by means of reference stars and then combining these to obtain (weighted) mean positions. This option is not likely to give best results here. The number of available reference stars is usually small. Also, the deep exposure frames have overexposed images of the reference stars and an iterative process involving tertiary reference stars' $\alpha, \delta$ then is required, starting from the short exposure frames.

Combining the $x, y$ data first is feasible without loss in accuracy due to the small field of view, the simple transformation geometry required here, and the large overlap in area of $\approx 40$ to $100 \%$ for all our CCD frames of a field. Our software allows for a rigorous correction for refraction, but this effect was found to be negligible for our data. A significant third-order optical distortion term was removed before the weighted least squares adjustment with a linear transformation model for overlapping frames. Weights were obtained from the precision of the image profile fits, which strongly depend on the magnitude of the stars. In addition a constant variance per frame was added to account for atmospheric effects, scaled by the inverse exposure time of the CCD frames. This approach allows using simultaneously more reference stars in a larger field of view in cases when mosaic CCD frames are available, and at the same time combines all measurable images of long and short exposure frames into a common system.

\subsection{Adjustment to Reference Star Positions}

Our standard plate reduction software package, as part of HBAPP (Hamburg Block Adjustment Program Package, Zacharias 1987), was used for the unweighted least-squares 
adjustment of the $x, y$ data to the reference star positions. The precision of the secondary reference stars contributes the largest part of the errors in this adjustment. The differences in the precision of the CCD $x, y$ data, e.g. due to the dependence on magnitude or the atmospheric effects as a function of exposure time, are not relevant here. Experiments with weights obtained from the astrograph reductions did not reveal any significant improvement.

Several pilot investigations were run to determine the most realistic mapping function of the telescopes (plate model). A linear plate model was finally adopted for routine processing with third-order optical distortion corrected prior to the adjustment.

\subsection{External Comparisons}

Field, as well as magnitude-dependent, systematic errors can be investigated externally by comparing $\alpha, \delta$ coordinates obtained from our CCD frames with positions obtained from a different telescope. For some fields ESO (European Southern Observatory) Schmidt plates, Lick Astrograph plates or prime focus plates from other telescopes are available.

Due to the high precision of our CCD data a comparison with Schmidt plates only reveals systematic errors in those plates. Positions obtained from prime focus plates depend on the same secondary reference stars of that field already used for the CCD reductions. Thus the Lick Astrograph offers the most promising external comparison. Plate measuring is in progess and results will be published in an upcoming paper. Here only a comparison between different KPNO and CTIO runs will be made.

A comparison of the optical positions with the quasi-error-free radio position can also

reveal systematic errors. But our aim is to calibrate the optical data independently of that comparison in order to draw astrophysically significant conclusions. 


\section{Results}

Because no photometric observations were obtained, all magnitudes in this paper are instrumental with the zeropoint adjusted to the mean photographic $\mathrm{V}$ magnitudes of the secondary reference stars.

\subsection{Internal $x, y$ Precision}

In this section we will compare the astrometric performance of various image profile fit models used to obtain $x, y$ coordinates from the pixel data of the CCD frames.

Initial tests with the 1-dimensional center of mass algorithm showed significantly larger errors in positions as compared to other algorithms and thus was not further investigated. We compared the 1-dimensional gaussian fit (1DG) and the allstar point spread function fit (ALS) from the DAOPHOT/IRAF package, as well as the 2-dimensional circular gaussian fit (2DG) from our astrometric software package (SAAC).

All comparisons were made only with stars appearing on all lists of $x, y$ coordinates obtained for the different profile fit models per frame, thus a unique limiting magnitude was used. All $x, y$ data were corrected for third-order optical distortion by applying the same value for the distortion coefficient (D3) and the location of the optical axis on the frame $\left(x_{0}, y_{0}\right)$ to all frames prior to the transformation with a linear model.

For routine reduction of the CCD frames in order to derive positions of extragalactic sources, the 2-dimensional circular gaussian (2DG) was adopted as our standard fit model because of its superior performance with respect to random, as well as systematic, errors as will be explained in the following two subsections. 


\subsubsection{KPNO $0.9 \mathrm{~m}$ Telescope}

Table 3 shows some results for the KPNO field of the source 1656+053. The CCD frames with internal numbers 68 and 74 are long exposures (300 sec), while all others are

short exposures (40 sec). Frames 68,74 and 73 are centered on the QSO radio source, while all others are part of a mosaic with offsets of 500" (735 pixel) in each coordinate. The seeing for all frames was $F W H M \approx 2.5$ pixel.

Most of the $x, y$ data obtained from the 1DG fit show a significant magnitude-dependent error in both coordinates for the faint stars as compared to the $x, y$ positions from the same CCD frame obtained by the other fit models. An example is shown in Figure 1 a) for the $\mathrm{x}$ coordinate of frame 73 in the comparison of fit model 2DG - 1DG. The other coordinate, as well as the data from other frames, looks similar.

The average position difference, as obtained by different profile fit models for the same pixel data, is on the order of 0.01 to 0.03 pixel, which is about 7 to 20 mas for the KPNO telescope. This is clearly a function of magnitude, increasing to the faint end. Figure 2 a) shows an example of the fit precision, $\sigma_{\text {fit }}$, as a function of magnitude for the $2 \mathrm{DG}$ fit of the pixel data obtained at KPNO from a 300-second exposure CCD frame of the field $0906+015$. The saturation limit for this frame is at $\approx 13.0^{m}$. A best positional precision of 0.01 pixel is found for star images in the magnitude range from the saturation limit to 3 magnitudes below. For faint stars the fit precision decreases sharply. Outliers have been visually inspected on the CCD frame and only galaxies and close double stars have been found to cause significantly larger fit errors than the mean for that magnitude, providing a good method for detecting non-stellar images.

Positions from multiple CCD frames of the same exposure time and the same field center were compared next. The standard error $\sigma_{x y}$ of such a frame-to-frame position transformation includes, besides the fit error $\sigma_{f i t}$, also errors introduced by the atmospheric 
turbulence $\sigma_{a t m}$. Figure 3 a) shows an example of $\sigma_{x}$ vs. magnitude for 2 frames from the field 0906+015 with a 300-second exposure time each. The limiting precision of $\approx 5$ mas can entirely be accounted for by the turbulence in the atmosphere. According to Lindegren (1980) we have for our case $\sigma_{a t m} \approx 10$ mas. Compared to the previous figure either $\sigma_{f i t}$

or $\sigma_{a t m}$ or both are overestimated. An explanation for overestimating $\sigma_{f i t}$ for bright stars is the difference of the observed image profile as compared to the assumed model. On the other hand, significant nightly variations of $\sigma_{a t m}$ are also well known.

Positions obtained for the brighter stars have been found to be less dependent on the profile fit algorithm used than those obtained for fainter stars. There was no systematic radial difference vs. radius found in any fit model comparisons of the same KPNO CCD frame.

The transformation of $x, y$ coordinates of the frames with short exposure times show a larger sigma than those of long exposure times for all fit models (Table 3) because of the noise added by the atmosphere. In comparing the performance of the different fit models, the 2DG shows the smallest random errors for the KPNO frames.

\subsubsection{CTIO $0.9 \mathrm{~m}$ Telescope}

Table 4 shows some of the results for the CTIO telescope for the field 0646-306. Frames 53 and 54 are long exposures (600 sec, $300 \mathrm{sec}$ ), while all others are short exposures (40 sec). Frames 53, 54 and 57 are centered on the QSO, while all others are part of a mosaic with offsets of $240 "=600$ pixel in both coordinates. Frame 53 has the poorest image quality as compared to the other frames.

Contrary to the results obtained with the KPNO telescope, here the simple 1DG fit is in good agreement with the 2DG fit. An example is shown in Figure $1 \mathrm{~b}$ ) for the difference 
in $\mathrm{x}$-coordinates vs. magnitude for frame 57. No magnitude-dependent systematic errors were found in the test field. The CTIO telescope has better sampling and with FWHM $\approx 3.8 p x$ in this test field there is a sufficient large number of pixels for both algorithms to determine consistent positions over a dynamic range of almost 8 magnitudes.

With the CTIO data the DAOPHOT allstar algorithm show small magnitudedependent systematic differences $(\approx 10 \mathrm{mas} / \mathrm{mag}$ ) as compared to the 2DG and $1 \mathrm{DG}$ fit results as well as $\approx 20$ mas systematic differences as a function of position in the field, when used with a single average point spread function (PSF) for the entire field of view. Figure 4 shows an example for the radial difference (2DG-ALS) vs. radius in frame 57. Clearly visible slightly elongated images at the edges of many CTIO frames require field-dependent PSF's to be used in DAOPHOT in order to obtain better results. The difficulty is how to relate these PSF's to each other astrometrically in order to get $x, y$ coordinates in a unique system for all stars in that frame on the 0.01 pixel level.

In the frame-to-frame comparison again the 2DG shows the best results as judged from the standard deviations of the transformations of the $x, y$ data. The 1DG algorithm performs nearly as good as the 2DG in this respect, while the ALS is clearly inferior.

For comparison with the KPNO results, Figure $2 \mathrm{~b}$ ) shows a plot of the fit precision vs. magnitude. The limiting precision here is only $\approx 0.02$ pixel. This can be explained by the poorer image quality of the CTIO as compared to the KPNO telescope, with variable deviations from the circular symmetric gaussian image profile depending on the location in the field. Expressed in arcseconds, both telescopes perform to about the same level of precision due to the better scale of the CTIO telescope. Figure 3 b) shows a frame-to-frame comparison, $\sigma_{x}$ vs. magnitude; both frames have been exposed for 600 seconds. Again a limit of $\approx 5$ mas in precision is reached for bright stars in a single exposure. The atmosphere seems to be the limiting factor. 


\subsection{Basic Mapping Model}

In this chapter the appropriate mapping model between the measured $x, y$ coordinates of the CCD frames and the corresponding standard coordinates $(\xi, \eta)$ will be investigated.

In order to allow for possible differences in scale and non-orthogonality of the axis a full linear transformation was adopted as our basic model. With orthogonal and non-orthogonal terms separated we have

$$
\begin{gathered}
\xi=a x+b y+c+e x+f y \\
\eta=-b x+a y+d+e y-f x
\end{gathered}
$$

\subsubsection{Optical Distortion Coefficient}

A third-order optical distortion term (D3) was determined from $x, y$ data of mosaic frames. A conventional plate adjustment $(\mathrm{CPA})$ of even a field with many $(\approx 20)$ reference stars revealed no significant D3 term. The mean error on the D3 term is approximately $1.0 \times 10^{-9} " / " 3$. In order to obtain a reliable value for the D3 term a procedure similar to that of the AGK 2 catalog project (Schorr \& Kohlschütter, 1951) was followed without the need for reference stars.

Pairs of overlapping CCD frames with offsets in their centers in the order of half a field size have been transformed onto each other by extending the linear model with the appropriate D3 term

$$
\begin{aligned}
& \Delta \xi=D 3\left(x_{1} r_{1}^{2}-x_{2} r_{2}^{2}\right) \\
& \Delta \eta=D 3\left(y_{1} r_{1}^{2}-y_{2} r_{2}^{2}\right)
\end{aligned}
$$


with $x_{1}, y_{1}$ and $x_{2}, y_{2}$ being the measured coordinates with respect to the center of distortion on frame 1 and frame 2 respecively and $r_{i}^{2}=x_{i}^{2}+y_{i}^{2}, i=1,2$. This algorithm assumes a common distortion term D3 for both frames, which is very realistic for frames taken shortly after each other with the same instrument and roughly the same location in the sky. This assumption was verified by comparing results for D3 obtained from various frame pairs.

The mean values for D3 and their errors for both telescopes are given in Table 5 along with the maximal effect per coordinate on these $2 \mathrm{~K}$ CCD's. The D3 term is highly significant and can be determined very precisely by this method. For convenience, conversion factors between the different units for quadratic and third-order terms in the CPA process are given in Table 6.

\subsubsection{Optical Distortion Center}

A significant offset of the center of distortion (optical axis) with respect to the geometric center of the CCD frame of $250 \pm 50$ pixel was found for the CTIO telescope in observing run 4 . A similar offset of $\approx 100 \pm 70$ pixel in another direction was found for the same telescope in observing run 3, while no such offsets were found for the KPNO telescope (observing runs 1,2). Figure 5 a) shows a vector plot of average differences (run 4 - run 3) of $x, y$ data of field 0743-006 with optical distortion applied at the geometric frame centers prior to combining the $x, y$ data of frames for each run. Figure $5 \mathrm{~b}$ ) shows the corresponding plot with optical distortion applied with respect to the optical axis as determined in a pilot investigation. In Figure 5 a) there is a systematic error of $\approx 70$ mas at the frame center which would have affected all source positions of that observing run. According to CTIO staff, such an offset of $\approx 200$ pixel is within the collimation tolerances of the instrument setup for each new observing run. 


\subsubsection{Tilt Terms}

A difference in tangential points of two overlapping frames causes tilt terms (p,q) of the form (König, 1933)

$$
\begin{aligned}
& \Delta \xi=p x^{2}+q x y \\
& \Delta \eta=p x y+q y^{2}
\end{aligned}
$$

Similar terms arise when individual frames are not perpendicular to the optical axis or the location of the tangential point is uncertain.

A maximum difference in the location of tangential points for overlapping mosaic

frames of 10 arcminutes was used here. This results in p,q terms as large as $1.4 \times 10^{-8 " ~ / " ~} 2$ (see Table 6). A maximum effect of $\Delta x \approx \Delta y \approx 0.01$ pixel $\leq 7$ mas is thus predicted for the edge of the field of view, which is totally negligible. Even assuming a tilt of the CCD plane with respect to the focal plane of the telescope (e.g. due to misalignment of the CCD camera) of $1^{\circ}$ results in a maximum effect of 0.06 pixel at the edge of the CCD frame, which would have little effect on the CPA results.

As expected, no significant $\mathrm{p}, \mathrm{q}$ terms were found neither in the $x, y$ transformation of overlapping plates, nor in the CPA of selected fields. A CPA with typical secondary reference stars is about a factor of 100 less sensitive to detect $\mathrm{p}, \mathrm{q}$ terms as is the $x, y$ data transformation of overlapping frames.

\subsection{External Calibration}

Both instruments show field-dependent systematic errors of the order of $\approx 20$ mas after applying the basic mapping model including third-order optical distortion (see e.g. 
Figure 5b).

Unfortunately, presently no external calibration with respect to a precise reference star catalog can be made. Plates were taken at the Lick Astrograph with very small epoch difference (2 months) from some CCD observing runs. When Hipparcos results will become available, a position catalog to $\approx 30$ mas precision for individual stars in an area of a few square degrees and down to 15th magnitude can be constructed for these external calibrations of our CCD data. A rigorous calibration of these FDP's (field distortion patterns) will then be possible, similar to the procedure used in photographic astrometry (Zacharias, 1995).

\subsection{Optical Positions of Reference Frame Sources}

Here we present position results for 16 sources, selected as a representative subset of all optical counterparts on the current list of candidates ( $>400$ sources) for the extragalactic radio-optical reference frame. The sources were selected from all 4 CCD observing runs, sampling all areas in the sky as well as a wide range in magnitudes. Problematic cases, e.g. close doubles and sparce fields were prefered in order to challenge the reduction technique. Sources with multiple observations (different observing runs) as well as fields with more than one set of seconday reference stars were selected in order to obtain accuracy estimates by external comparisons.

Table 7 gives a summary of the results. The positions are in the FK5/J2000 system as represented by the IRS catalog, and based on the 2-dimensional circular gaussian (2DG) fit model. The radio positions are taken from our radio reference frame (Johnston et al. 1995). The rms difference (radio-optical) is $\approx 100$ mas for $\Delta \alpha \cos (\delta)$ and $\Delta \delta$. Compared to the expected internal errors this only shows the "wobbles" of the current optical system 
and gives no insight into the accuracy of the CCD observations.

A reduction based on a Hipparcos intermediate solution was performed for the Hipparcos Working Group on Reference Link and shows an external error consistent with our error estimations. Detailed results will be published when the final Hipparcos catalog becomes available.

\subsection{Remarks on Individual Sources}

The following sources are optical doubles, most likely due to a foreground star. The images of both the extragalactic source and the companion are consistent with a stellar profile in all cases. No extended structure, e.g. of an underlying galaxy, was detected. All these sources are suitable for the radio-optical reference frame link, at least for now at the 30 mas level.

$0153+744$ is an optical double with a separation of $\approx 7 \operatorname{arcsec}(10$ pixel $)$, which could be resolved without any problems.

0605-085 is an optical double with a flux of the companion 5 times brighter than the extragalactic source and a separation of $3 \operatorname{arcsec}(4.5 \mathrm{px}$ at KPNO, 7 px at CTIO). A subtraction of the image profile of the companion was required to obtain a position of the extragalactic source.

0607-157 is an optical double with a companion 2 times weaker than the extragalactic source with a separation of $4 \operatorname{arcsec}(6 \mathrm{px}$ at KPNO, $10 \mathrm{px}$ at CTIO). The cut-out and fit-up procedures (see Section 4) allowed consistent image profile fits.

0743-006 is an optical double with a companion about a factor of 4 weaker than the extragalactic source and separated by $2.7 \operatorname{arcsec}(6 \mathrm{px}$ at CTIO). The fit-up and cut-out 
procedures with the 2DG fit gave consistent positions within 10 mas, while the allstar result was different by $\approx 30$ mas. Figure 6 shows a contour plot of this source, obtained from a 200-second exposure CTIO CCD frame in 1.5 arcsec seeing (3.7 pixel FWHM).

$1800+440$ is an optical double (3 arcsec) which was successfully fitted with the allstar algorithm as well as with the 2DG with use of cut-out and fit-up procedures. A contour plot of this source has been shown elsewhere (Zacharias et al. 1995).

The large number of optical doubles (5 out of 16 sources) presented here is not representative for all observations. These objects were selected for this pilot investigation.

\subsection{Comparison of Multiple Data Sets}

The sources 0336-019, 0605-085, 0607-157 and 0743-006 have been observed in more than one observing run. The mean quadratic difference in positions of the same source (see Table 7) obtained from different runs is $\approx 25$ mas, showing the high accuracy of the CCD observations. For 0743-006 there are 2 sets of secondary reference stars available from the Hamburg and Black Birch astrographs respectively. The agreement between the sets of secondary reference stars is on the same $\approx 25$ mas level, indicating the high accuracy of the secondary reference stars and the successful control of possible magnitude-dependent systematic errors in the astrograph fields.

\section{Discussion}

\subsection{Astrometric Properties of Both Telescopes}

Based on the results of the previous section we estimate some individual random and systematic error contributions for observations made with both telescopes. 
The error of a single $x, y$ observation depends on the magnitude of the object. For faint objects the photon statistics limit the precision and the internal error obtained from the profile fit or the $x, y$ transformation from frame to frame is a good estimate of the accuracy of the position. For bright objects the random errors are as low as $\approx 0.01$ pixel and the systematic errors dominate. The astrometrically usable dynamic range depends on the pixel scale (positions for faint stars are getting worse when undersampled), as well as on the sky background level. Although most of our candidates are bright enough to be observed successfully within the full-Moon period, this has compromised the usable dynamic range, stressing the importance of the additional short exposure frames.

Depending on the magnitude of the extragalactic reference link sources, a typical value for the random error of an $x, y$ coordinate of a single image on a CCD frame is $\sigma_{x y} \approx 15$ mas, but the range was found to be as large as 5 to 31 mas (see Table 7). Systematic errors as a function of magnitude are expected to be $\leq 10$ mas over the magnitude range from secondary reference stars to extragalactic objects when using the appropriate profile fit model (2DG). Systematic errors as a function of location on the CCD frame are found on the $\approx 20$ mas level, being larger far from the optical axis but negligible at the frame centers, where the image of the extragalactic object is usually located. These systematic errors will average out for different fields because of the different location of reference stars in each field. A more rigorous calibration of these FDP's is in progress which needs to be performed for each observing run separately, at least for the CTIO telescope.

\subsection{Accuracy Estimate for the Link Procedure}

In this section we will combine all estimates of individual error contributions for the optical observations of the reference frame link procedure based on counterparts of extragalactic radio sources. Special consideration is given to systematic errors in this 
multi-step procedure.

We will refer to step 1 as the primary optical reference system. Options considered here are the IRS and the future Hipparcos and Tycho catalogs. By step 2 we denote the secondary reference stars which usually are obtained photographically by wide-field astrographs. An intermediate step 3 is sometimes taken with tertiary reference stars obtained either by Schmidt telesopes or CCD observations with wide fields. The last step is always the optical observation of the radio source counterpart itself, either photographically or with CCD.

All error estimates given here are approximate. The aim is to identify the largest error contribution, to compare the performance of different options and to find the limits of this approach to the radio-optical reference frame link procedure. All formulae and values to follow are for one coordinate.

As can be seen from the results in Table 7 , the standard error of unit weight $\sigma_{C P A}$ of the adjustment of $\operatorname{CCD} x, y$ to secondary reference star positions can be as low as $\approx 45$ mas, indicating a sub-micrometer accuracy from a single astrograph plate. Values for $\sigma_{C P A}$ increase with epoch difference of the secondary reference star observations and the CCD observations. This is due to the unknown proper motions in the secondary reference star data. No systematic corrections (e.g. galactic rotation) were applied here.

\subsubsection{Algorithm}

First we will define some quantities to be used in the link of step $i$ to step $i+1$.

Let $n_{i}$ be the number of stars to be used as reference stars to link step $i$ to step $i+1$. The random error of such a link star in step $i$ we denote with $\sigma r a n_{i}$, and $\sigma s y s_{i+1}$ is the systematic error for the link of step $i$ to $i+1$. Similarly, $\sigma x y_{i+1}$ is the precision (random 
error) of a single $x, y$ observation for a link star in step $i+1$, and $m_{i+1}$ is the number of observations (exposures) for each link star in step $i+1$.

The random error of a star position obtained in step $i+1$ then is approximately

$$
\operatorname{\sigma ran}_{i+1}=\frac{\sigma x y_{i+1}}{\sqrt{m_{i+1}}}
$$

Because there is only a limited number of link stars with associated errors between the two steps, the link of the system of step $i$ and $i+1$ can not be made error-free. The uncertainty in the zeropoint offset, $\sigma z_{i+1}$, between the coordinate systems in step $i$ and $i+1$ is approximately

$$
\sigma z_{i+1}=\sqrt{\frac{\sigma^{2} \operatorname{ran}_{i}+\sigma^{2} \operatorname{ran}_{i+1}}{n_{i}}}=\frac{\sigma_{C P A}}{\sqrt{n_{i}}}
$$

Here $\sigma_{C P A}$ is the standard error of unit weight in the least-squares adjustment of combined $x, y$ data of step $\mathrm{i}+1$ to reference star data of step i. But these formulae hold only for the central area of a frame (plate) and for a simple (linear) mapping model. In addition, a factor larger than one is required for other cases, and a rigorous derivation is given by Eichhorn \& Williams (1963). Our zeropoint offset can be considered as a special case of the error contribution due to error progapation of the plate constants to field star positions.

Finally, the accuracy of a position of an extragalactic link source, $\sigma_{Q}$, is approximately the rms sum of all zero point offsets from previous steps plus the systematic errors and the precision, $\operatorname{\sigma ran}_{Q}$, of all optical observations of the source itself

$$
\sigma_{Q}=\sqrt{\sigma^{2} \operatorname{ran}_{Q}+\sum_{i=2}^{k} \sigma^{2} z_{i}+\sum_{i=2}^{k} \sigma^{2} s y s_{i}}
$$

With systematic errors we mean here errors not averaging out with the number of 
stars used for the link of a single extragalactic source. It is assumed that such systematic errors (e.g. depending on magnitude for a particular plate) will be different for different extragalactic source fields and thus (at least partly) random when results for many sources are combined. Systematic errors inherent in this technique and not averaging out with different fields can't be investigated here. An external comparison with other methods for the extragalactic link procedure will be made in the future.

\subsubsection{Accuracy Estimate for the Secondary Reference Star Positions}

Here we will start out with 3 options for the primary optical reference system (step 1) and discuss 12 cases for determining secondary reference star positions (step 2). All cases are summarized in Table 8.

The currently available IRS system has a density of $\approx 0.9$ stars $/$ degree $^{2}$ and a precision of $\approx 200$ mas for epochs of 1980 to 1994 , where most of our data were taken. The usable field of views for the Hamburg Zone Astrograph (ZA), the Black Birch Astrometric Observatory (BBAO) astrograph and the Lick Astrograph are approximately 36, 25 and 9 degree $^{2}$ respectively. For the Hipparcos catalog we assume a mean $\sigma_{r a n}=10$ mas for the epoch range of our data. After the Tycho catalog is combined with the Astrographic Catalog (AC) data in order to obtain proper motions, we assume a mean $\operatorname{\sigma ran}_{1}=50$ mas for that catalog at the epoch of our data.

Systematic errors depending on magnitude are controlled with a diffraction grating at the astrographs. Preliminary results indicate magnitude terms on the order of 0 to $1 \mu \mathrm{m}$ per 5 magnitudes, this is up to 20 mas/mag. The error on determining this term is about 2 mas/mag. This is a systematic error for a plate or field, which varies from field to field.

All cases 4 are based on a $1 d e g^{2}$ CCD frame. Cases $4 \mathrm{~b}$ and $4 \mathrm{c}$ assume a mini-block 
adjustment of an area of $\approx 9 \mathrm{deg}^{2}$. As can be seen from Table 8, a considerable improvement will be gained from the Hipparcos catalog as compared to the current IRS. A further improvement can be obtained by using the Tycho catalog, but this means a tremendous effort on plate measuring and the availability of the $\mathrm{AC}$ in order to derive good proper motions to be combined with the original Tycho observations. This is only worth the effort if the systematic errors can be controlled to this level.

The CCD option is only competitive here when used with block adjustment techniques, at least in local fields. Because of the expected lower systematic errors, e.g. as a function of magnitude, this may be the way to go in the future. Such CCD observations could be based directly on Hipparcos stars with about the same precision as could be obtained from a Tycho-based solution, thus excluding possible systematic errors from the Tycho proper motions.

\subsubsection{Position Accuracy of the Extragalactic Sources}

With the algorithm as above and a typical internal precision of a CCD observation of $\sigma x y \approx 15$ mas and $m=2$ observations per source, we have a random error for the optical position of an extragalactic source of $\operatorname{\sigma ran}_{Q} \approx 11$ mas.

Random errors from the CCD observations of the link stars (secondary reference stars) are even smaller due to their brightness, a typical value is $\sigma_{f i t} \leq 10$ mas. The largest error contribution here is the influence due to the atmosphere in case of the short exposure frames. According to Lindegren (1980) this amounts to $\sigma x y_{3} \approx 30$ mas for a single exposure; with $m_{3} \approx 4$ we have according to Eq. (1) $\operatorname{\sigma ran}_{3} \approx 15$ mas.

Let us assume a random error from the astrograph observations of a link star (secondary reference star) of $\operatorname{\sigma ran}_{2} \approx 70$ mas. With $n_{2} \approx 15$ stars for that link between step 2 
(secondary reference stars) and step 3 (CCD observations) we thus obtain according to Eq. (2) a zeropoint error of $\sigma z_{3} \approx 20$ mas. This strongly depends on the number of stars used and the epoch difference between the CCD and astrograph observations. Individual results of the precision and accuracy properties of the CCD observations can be found in Table 7 .

Putting everything together, and assuming $\sigma s y s_{2} \approx \sigma s y s_{3} \approx 10$ mas, we expect an accuracy of the optical position of an extragalactic object to be in the order of $\sigma_{Q} \approx 30$ mas plus $\sigma z_{2}$ as discussed in the previous section, which is negligible in case of Hipparcos-catalog-based secondary reference stars $\left(\sigma z_{2} \approx 5\right.$ mas). When using the IRS catalog we have $\sigma z_{2} \approx 45$ mas plus $\sigma s y s_{1} \approx 100$ mas. Individual estimates of $\sigma_{Q}$ (based on the Hipparcos catalog) are given in Table 7 for each object. Thus currently the largest error contribution comes from the primary system, the FK5, as represented by the IRS. With the use of the Hipparcos results the largest error contribution comes from the weak link of the secondary reference stars to the CCD observations due to the small field of view of the CCD's and the relatively poor limiting magnitude of the secondary reference stars. A large epoch difference between the secondary reference star and the QSO observations significantly increases the noise in this crucial step, regardless of any additional possible systematic errors indroduced by unknown proper motions.

\subsection{Comparison to Other Investigations}

Other major procedures for the position link of the radio and optical reference frame are the HST (Hubble Space Telescope) observations of selected pairs of Hipparcos stars and bright extragalactic candidates and the VLBI/VLA observations of Hipparcos radio stars. The HST observations are of higher internal precision than our observations but are not so

numerous ( $\approx 40$ pairs) and depend on the absolute calibration of the FGS fields. The radio star approch is very precise and direct but is based only on less than 10 objects which are 
not well distributed over the sky.

Our approach contributes significantly to the link process and allows the important check to be made on possible systematic errors of the other methods.

The Sloan Digital Sky Survey (SDSS) will be helpful in order to densify the grid of secondary (and tertiary) reference stars in the galactic north pole region. The positional accuracy of the optical counterparts of the extragalactic radio sources from SDSS will be inferior to our observations due to shorter integration time.

\section{Conclusions}

The feasibility of this approach to the radio-optical reference frame link has been proven here using wide field CCD observations with the KPNO and CTIO $0.9 \mathrm{~m}$ telescopes.

The link to the primary reference star system, as represented by the Hipparcos catalog in the near future, is based entirely on photographic plates obtained with dedicated astrographs in both hemispheres. A deeper limiting magnitude and higher precision for the link stars is most important now, and CCD observations at the astrographs are in progress to provide more reference star positions. These observations will also provide an additional determination of possible magnitude-dependent systematic errors in the entire procedure.

The $0.9 \mathrm{~m}$ telescope CCD observations have acquired a huge amount of high precision optical observations of extragalactic sources within a short period of time. The precision for a single long exposure is in the range of 5 to 31 mas (average $\approx 15$ mas) depending on the magnitude of the object. Field-dependent systematic errors exist on a 20 mas level, but they will be externally calibrated in the near future. A 20 mas precision level was reached previously with prime focus photography, but only for few objects per year at large telescopes. 
With 3 more CCD runs we hope to complete observations at the $0.9 \mathrm{~m}$ telescopes for about 400 sources which would allow a position tie to the Hipparcos system on the 1 mas level.

In addition to the position information, a structure analysis of the optical counterparts is highly desirable. Because these objects have already been selected to be compact, the search for structure has to be made with much higher resolution than the $0.9 \mathrm{~m}$ ground-based telescopes can offer. Optical interferometry, adaptive optics or the HST are the only options at the moment. Currently our structure analysis is limited to identify suitable candidates for the link process, i.e. optical sources free of nearby disturbing foreground stars and galaxies.

After a sufficiently rigid link between the radio and optical systems has been established, our observations will be used to identify outliers which will have astrophysical implications about the nature of these compact extragalactic objects. The biggest advantage of our approach to the extragalactic reference frame link is the large number of sources involved. Observations can easily be maintained in the future from ground-based telescopes, providing also an epoch difference large enough for a proper motion tie of the Hipparcos system within the next decade. With only minor improvements and more observations a much higher precision and accuracy can be reached by this technique in the near future.

Position results of a large number of sources will be published after the Hipparcos catalog becomes available. No conclusions should be drawn from the positions published here based on the IRS system. When using a Hipparcos intermediate solution, as required for the Hipparcos Working Group on Reference Link, positional results with respect to the radio frame are in agreement with the error estimation given above.

Ultimately a space mission like FAME (Johnston 1995) or GAIA (Perryman \& van Leeuwen 1996) will provide optical positions for some (in case of FAME) or most (in case 
of GAIA) of these objects with an accuracy better than current VLBI radio observations. Until then we will hopefully have a much better understanding of the astrophysical and astrometric properties of these objects in order to be able to concentrate on the most suitable candidates for a reference frame.

Chr. de Vegt wishes to thank the Bundesministerium für Forschung und Technologie (BMFT) for financial support under Grant No. 50008810 (Hipparcos). We further thank J.L. Russell and M.I. Zacharias for assistance with observing, as well as J. Münkel for assistance with the astrographic plate measuring and reduction process. 


\section{REFERENCES}

Corbin,T.E. \& Warren,W.H., 1991, International Reference Stars Catalog, NASA, NSSDC $91-11$

Corbin,T.E. \& Urban,,S.E. 1990, in Inertial Coordinate Systems on the Sky, IAU Symp. No. 141, Kluwer, Dordrecht, p. 433

de Vegt,C., 1978, Bull. CDS 15, 51

de Vegt,C., Schramm,J. \& Johnston,K.J., 1987, AJ93, 261

Eichhorn, H. \& Williams, C.A. 1963, AJ68, 221

IAU Inf. Bull. No. 74, 1995, Kluwer, Dordrecht, p. 3

Johnston,K.J., Fey,A.L., Zacharias,N., Russell,J.L., Ma,C., de Vegt,C., Reynolds,J.E., Jauncey,D.L., Archinal,B.A., Cater,M.S., Corbin,T.E., Eubanks,T.M., Florkowski,D.R., Hall,D.M., McCarthy,D.D., McCulloch,P.M., King,E.A., Nicolson,G.D. \& Shaffer,D.B., 1995, A Radio Reference Frame, accepted by AJ

Johnston, K.J. (principal investigator) 1995, Proposal for the FAME space mission, U.S. Naval Observatory, Washington DC

König, A. 1933, Handbuch der Astrophysik, Vol.I, part 1, J.Springer, Berlin

Lindegren, L. 1980, A\&A89, 41

Perryman,M.A.C. \& van Leeuwen,F. (eds.) 1996, Future Possibilities for Astrometry in Space, proceedings of a workshop at RGO, Cambridge, England, European Space Agency (ESA) publication, in press

Routly,P.M., 1983, in Sky with Ocean Joined, Proc. of the Sesquicentennial Symp., U.S. Naval Observatory, p. 145

Schorr, R. \& Kohlschütter, A., 1951, Zweiter Katalog der Astronomischen Gesellschaft, Vol. I, p. E9, Hamburg Observatory, Hamburg-Bergedorf 
Schramm, J. 1988 Ph.D.Thesis, University of Hamburg

Stetson,P.B., 1987, PASP, 99, 191

Turon,C. et al., 1992, Hipparcos Input Catalog, ESA SP-1136, European Space Agency, Vols. $1-7$

Winter, L., de Vegt, C., Steinbach, M., Zacharias, N. 1992 in Digitised Optical Sky Surveys, edited by H.T.MacGillivray \& E.B.Thomson, ASSL proceedings series Vol. 174 (Edinburgh), Kluwer Acad. Publisher, Dordrecht, p.123

Winter, L., 1994, in IAU Symp. No. 161, Astronomy from wide-field imaging, edited by H.T.MacGillivray, E.B.Thomson, B.M.Lasker, I.N.Reid, D.F.Malin, R.M.West \& H.Lorenz, p. 288

Zacharias,N. 1987, PhD. thesis, University of Hamburg

Zacharias,N. 1995, AJ109, 1880

Zacharias, N. 1996, in preparation

Zacharias, N., de Vegt, C., Winter, L. \& Weneit, W., 1994, in IAU Symp. 161, Astronomy from wide-field imaging, edited by H.T.MacGillivray, E.B.Thomson, B.M.Lasker, I.N.Reid, D.F.Malin, R.M.West \& H.Lorenz, p. 285

Zacharias,N., Fey,A.L., Russell,J.L. \& Johnston,K.J., 1995, Proc. of IAU Symp. No. 166, The Hague, in press 
Fig. 1.- Difference in $\mathrm{x}$-coordinate for 2-dimensional minus 1-dimensional gaussian fit from the same pixel data vs. instrumental magnitude, example for a) KPNO, b) CTIO. 1 dot represents the mean of 4 differences.

Fig. 2.- Fit error for $\mathrm{x}$-coordinate of individual stars vs. instrumental magnitude; example for a) KPNO, b) CTIO.

Fig. 3.- Standard error of unit weight for $\mathrm{x}-$ coordinate of a frame-to-frame transformation with the same tangential point vs. instrumental magnitude; example for a) KPNO, b) CTIO. 1 dot represents the mean of 4 differences.

Fig. 4.- Radial difference for 2-dimensional gaussian fit minus point spread function fit from the same pixel data vs. radial distance from the CCD center; an example from CTIO data.

Fig. 5.- Vector plot for $x, y$ position differences of the field 0743-006 as observed in Dec.94 minus as observed in Febr.95 with the same CTIO telescope, with optical distortion corrected at a) the geometrical frame center, b) the assumed location of the optical axis. The differences are increased by a scale factor of 2000 .

Fig. 6.- Contour plot of 0743-006 from a 200 seconds CCD exposure taken at CTIO. The image of the QSO is the brighter of the two objects. 
Table 1: Properties of instruments (telecopes and CCD's).

\begin{tabular}{lccl} 
& KPNO & CTIO & \\
\hline aperture & 0.9 & 0.9 & meter \\
focal ratio & $\mathrm{f} / 7.5$ & $\mathrm{f} / 13.5$ & \\
optical system & $\mathrm{R} / \mathrm{C}+$ corr. & cassegrain & \\
plate scale & 28.3 & 17.0 & $\mathrm{n} / \mathrm{mm}$ \\
pixel size & 24.0 & 24.0 & $\mu \mathrm{m}$ \\
pixel scale & 0.68 & 0.40 & $" /$ pixel \\
field of view & 23.1 & 12.8 &, \\
readout time & 140 & 70 & seconds
\end{tabular}

Table 2: Summary of observing runs.

\begin{tabular}{lcccc} 
run number & 1 & 2 & 3 & 4 \\
\hline date & April 94 & Oct. 94 & Dec. 94 & Feb. 95 \\
observatory & KPNO & KPNO & CTIO & CTIO \\
number of usable nights & 5 & 5 & 7.5 & 8 \\
average seeing (arcsec) & $1.6 \ldots 3$ & $1.4 \ldots 1.8$ & $1.2 \ldots 1.8$ & $1.2 \ldots 2.0$ \\
number of observed sources & 35 & 70 & 60 & 68 \\
number of object frames & 103 & 163 & 117 & 150 \\
number of short exp. frames & 89 & 160 & 238 & 340 \\
number of test frames & 61 & 6 & 23 & 13
\end{tabular}


Table 3: Results from frame \& fit model to frame \& fit model comparisons of KPNO test field 1656+053. Standard errors $\sigma_{x}, \sigma_{y}$ are given in milli-pixel.

\begin{tabular}{|c|c|c|c|c|c|c|}
\hline 68 & $2 \mathrm{DG}$ & 68 & ALS & 22 & 23 & long exposure \\
\hline 68 & $2 \mathrm{DG}$ & 68 & $1 \mathrm{DG}$ & 28 & 24 & \\
\hline 74 & $2 \mathrm{DG}$ & 74 & ALS & 18 & 20 & long exposure \\
\hline 74 & $2 \mathrm{DG}$ & 74 & $1 \mathrm{DG}$ & 19 & 20 & \\
\hline 71 & $2 \mathrm{DG}$ & 71 & $1 \mathrm{DG}$ & 29 & 30 & magnitude equation \\
\hline 71 & $2 \mathrm{DG}$ & 71 & ALS & 39 & 36 & ok \\
\hline 72 & $2 \mathrm{DG}$ & 72 & $1 \mathrm{DG}$ & 29 & 24 & magnitude equation \\
\hline 72 & $2 \mathrm{DG}$ & 72 & ALS & 32 & 31 & ok \\
\hline 73 & $2 \mathrm{DG}$ & 73 & $1 \mathrm{DG}$ & 27 & 34 & magnitude equation \\
\hline 73 & $2 \mathrm{DG}$ & 73 & ALS & 20 & 22 & ok \\
\hline 68 & $2 \mathrm{DG}$ & 74 & $2 \mathrm{DG}$ & 35 & 35 & ok long - long \\
\hline 68 & $1 \mathrm{DG}$ & 74 & $1 \mathrm{DG}$ & 45 & 41 & \\
\hline 68 & ALS & 74 & ALS & 44 & 41 & \\
\hline 74 & $2 \mathrm{DG}$ & 73 & $2 \mathrm{DG}$ & 47 & 55 & ok long - short \\
\hline 74 & $1 \mathrm{DG}$ & 73 & $1 \mathrm{DG}$ & 72 & 72 & \\
\hline 74 & ALS & 73 & ALS & 63 & 75 & \\
\hline 73 & $2 \mathrm{DG}$ & 72 & $2 \mathrm{DG}$ & 62 & 44 & \\
\hline 73 & $1 \mathrm{DG}$ & 72 & $1 \mathrm{DG}$ & 70 & 55 & \\
\hline 73 & ALS & 72 & ALS & 78 & 61 & \\
\hline 73 & $2 \mathrm{DG}$ & 71 & $2 \mathrm{DG}$ & 64 & 73 & \\
\hline 73 & $1 \mathrm{DG}$ & 71 & $1 \mathrm{DG}$ & 73 & 77 & \\
\hline 73 & ALS & 71 & ALS & 84 & 81 & \\
\hline
\end{tabular}


Table 4: Results similar to Table 3 for the CTIO test field 0646 - 306.

\begin{tabular}{|c|c|c|c|c|c|c|}
\hline 54 & $2 \mathrm{DG}$ & 54 & $1 \mathrm{DG}$ & 30 & 36 & long exposure \\
\hline 54 & $2 \mathrm{DG}$ & 54 & ALS & 50 & 63 & \\
\hline 57 & $2 \mathrm{DG}$ & 57 & $1 \mathrm{DG}$ & 38 & 40 & short exposure \\
\hline 57 & $2 \mathrm{DG}$ & 57 & ALS & 39 & 58 & \\
\hline 58 & $2 \mathrm{DG}$ & 58 & $1 \mathrm{DG}$ & 31 & 37 & short exposure \\
\hline 58 & $2 \mathrm{DG}$ & 58 & ALS & 48 & 75 & \\
\hline 53 & $2 \mathrm{DG}$ & 54 & $2 \mathrm{DG}$ & 46 & 43 & long exposure \\
\hline 53 & $1 \mathrm{DG}$ & 54 & $1 \mathrm{DG}$ & 49 & 48 & \\
\hline 53 & ALS & 54 & ALS & 75 & 89 & fit model not ok \\
\hline 54 & $2 \mathrm{DG}$ & 57 & $2 \mathrm{DG}$ & 62 & 60 & \\
\hline 54 & $1 \mathrm{DG}$ & 57 & $1 \mathrm{DG}$ & 80 & 73 & \\
\hline 54 & ALS & 57 & ALS & 91 & 96 & \\
\hline 57 & $2 \mathrm{DG}$ & 58 & $2 \mathrm{DG}$ & 68 & 80 & \\
\hline 57 & $1 \mathrm{DG}$ & 58 & $1 \mathrm{DG}$ & 80 & 83 & \\
\hline 57 & ALS & 58 & ALS & 101 & 128 & \\
\hline 57 & $2 \mathrm{DG}$ & 60 & $2 \mathrm{DG}$ & 74 & 79 & \\
\hline 57 & $1 \mathrm{DG}$ & 60 & $1 \mathrm{DG}$ & 82 & 90 & \\
\hline 57 & ALS & 60 & ALS & 106 & 107 & \\
\hline
\end{tabular}


Table 5: Summary on third-order optical distortion of both telescopes

\begin{tabular}{lrrl} 
& KPNO & CTIO & unit \\
\hline mean value of D3 & 50 & 134 & $" / " 3$ \\
mean value of D3 & $-0.49 \times 10^{-9}$ & $-0.46 \times 10^{-9}$ & $p x / p x^{3}$ \\
standard error on D3 & $0.02 \times 10^{-9}$ & $0.03 \times 10^{-9}$ & $p x / p x^{3}$ \\
maximum effect at frame edge & 0.53 & $0.49 \mathrm{px}$ \\
scale & 0.68 & $0.40 " / \mathrm{px}$
\end{tabular}

Table 6: Conversion factors for quadratic and third-order terms, scale in arcsecond (") per pixel $(\mathrm{px})$ and $\mathrm{C} 1=3600 * 180 / \pi \approx 206264.8$

\begin{tabular}{|c|c|c|c|}
\hline term & input units & output units & factor \\
\hline quadratic $(\mathrm{p}, \mathrm{q})$ & $p x / p x^{2}$ & $" / " 2$ & $1 /$ scale \\
\hline quadratic (p,q) & $" /{ }^{2}$ & $\mathrm{rad} / \mathrm{rad}^{2}$ & $\mathrm{C} 1$ \\
\hline tilt angle $(\mathrm{p}, \mathrm{q})$ & $\mathrm{rad} / \mathrm{rad}^{2}$ & $"$ & $\mathrm{C} 1$ \\
\hline distortion (D3) & $p x / p x^{3}$ & $"{ }^{\prime 3}$ & $1 /$ scale $^{2}$ \\
\hline distortion (D3) & $"{ }^{3}$ & $\mathrm{rad} / \mathrm{rad}^{3}$ & $C 1^{2}$ \\
\hline distortion (D3) & $"{ }^{\prime}{ }^{3}$ & $" / d e g^{3}$ & $3600^{3}$ \\
\hline
\end{tabular}


Table 8: Accuracy of secondary reference stars. Additional errors due to unknown proper motions of the anonymous secondary reference stars are not included here. The Tycho catalog is assumed to have been complemented with ground-based observations (AC) in order to contain reliable proper motions. Case $4 \mathrm{~b}$ and 4c assume a block adjustment of overlapping frames in a $9 \mathrm{deg}^{2}$ area (mosaic CCD). See Section 6.2.1 for explanation of columns.

\begin{tabular}{|c|c|c|c|c|c|c|c|c|c|}
\hline case & $\begin{array}{l}\text { primary } \\
\text { catalog }\end{array}$ & $\begin{array}{l}\operatorname{\sigma ran}_{1} \\
\text { mas }\end{array}$ & $\begin{array}{r}\sigma s y s_{1} \\
\text { mas }\end{array}$ & instr. & $n_{1}$ & $\begin{array}{r}\sigma x y_{2} \\
\text { mas }\end{array}$ & $m_{2}$ & $\begin{array}{r}\sigma r a n_{2} \\
\operatorname{mas}\end{array}$ & $\begin{array}{r}\sigma z_{2} \\
\text { mas }\end{array}$ \\
\hline $1 \mathrm{a}$ & IRS & 200 & 100 & $\mathrm{ZA}$ & 32 & 90 & 4 & 45 & 36.0 \\
\hline $1 \mathrm{~b}$ & & & & $\mathrm{BBAO}$ & 22 & 100 & 4 & 50 & 44.0 \\
\hline $1 \mathrm{c}$ & & & & Lick & 8 & 50 & 3 & 29 & 71.0 \\
\hline $2 \mathrm{a}$ & Hip & 10 & 1 & $\mathrm{ZA}$ & 100 & 90 & 4 & 45 & 4.6 \\
\hline $2 \mathrm{~b}$ & & & & BBAO & 70 & 100 & 4 & 50 & 6.1 \\
\hline $2 \mathrm{c}$ & & & & Lick & 25 & 50 & 3 & 29 & 6.1 \\
\hline $3 a$ & Tycho & 50 & 5 & $\mathrm{ZA}$ & 1000 & 90 & 4 & 45 & 2.1 \\
\hline $3 \mathrm{~b}$ & & & & $\mathrm{BBAO}$ & 700 & 100 & 4 & 50 & 2.7 \\
\hline $3 \mathrm{c}$ & & & & Lick & 250 & 50 & 3 & 29 & 3.7 \\
\hline $4 \mathrm{a}$ & Tycho & 50 & 5 & CCD & 25 & 30 & 4 & 15 & 10.4 \\
\hline $4 \mathrm{~b}$ & & 50 & 5 & $\mathrm{mCCD}$ & 225 & 30 & 4 & 15 & 3.3 \\
\hline $4 \mathrm{c}$ & Hip & 10 & 1 & $\mathrm{mCCD}$ & 22 & 20 & 4 & 10 & 3.0 \\
\hline
\end{tabular}


Table 7. Position Results of Selected Sources

\begin{tabular}{|c|c|c|c|c|c|c|c|c|c|c|c|c|c|c|c|c|c|c|}
\hline \multirow[t]{2}{*}{ source } & \multirow[t]{2}{*}{$\mathrm{t}$} & \multirow[t]{2}{*}{ mag } & \multirow[t]{2}{*}{ run } & \multicolumn{2}{|c|}{$\mathrm{nCCD}$} & \multirow{2}{*}{$\begin{array}{c}\mathrm{FW} \\
\mathrm{px}\end{array}$} & \multirow{2}{*}{$\begin{array}{l}\sigma_{x y} \\
\text { mas }\end{array}$} & \multicolumn{3}{|c|}{ secondaries } & \multirow{2}{*}{$\begin{array}{r}\sigma_{C P A} \\
\operatorname{mas}\end{array}$} & \multicolumn{3}{|c|}{ RA (2000) } & \multicolumn{3}{|c|}{ DC (2000) } & \multirow[t]{2}{*}{ Or } \\
\hline & & & & 1 & $\mathrm{~s}$ & & & tel & $\mathrm{yr}$ & $n_{r e f}$ & & $\mathrm{~h}$ & $\mathrm{~m}$ & $\mathrm{~s}$ & $\circ$ & & & \\
\hline $0153+744$ & $\mathrm{Q}$ & 16 & 2 & 2 & 4 & 2.5 & 11 & $\mathrm{ZA}$ & 79.8 & 31 & 147 & 01 & 57 & 34.9189 & +74 & 42 & 43.221 & -1 \\
\hline $0308-611$ & $\mathrm{Q}$ & 18 & 3 & 2 & 6 & 3.4 & 9 & $\mathrm{BY}$ & 90.4 & $10 \mathrm{~m}$ & 82 & 03 & 09 & 56.1035 & -60 & 58 & 39.063 & \\
\hline 0336-019 & $\mathrm{Q}$ & 18.4 & 2 & 3 & 2 & 2.3 & 15 & $\mathrm{BY}$ & 89.9 & 13 & 90 & 03 & 39 & 30.9368 & -01 & 46 & 35.772 & - \\
\hline 0336-019 & & & 3 & 3 & 0 & 3.8 & 8 & BY & 89.9 & 6 & 62 & 03 & 39 & 30.9341 & -01 & 46 & 35.772 & - \\
\hline $0605-085$ & $\mathrm{Q}$ & 18.5 & 2 & 3 & 2 & 2.3 & 17 & BY & 91.2 & 23 & 63 & 06 & 07 & 59.6832 & -08 & 34 & 50.138 & -2 \\
\hline $0605-085$ & & & 3 & 4 & 5 & 4.0 & 16 & BY & 91.2 & $13 \mathrm{~m}$ & 69 & 06 & 07 & 59.6809 & -08 & 34 & 50.111 & -2 \\
\hline $0607-157$ & $\mathrm{Q}$ & 18 & 2 & 2 & 2 & 2.4 & 9 & BY & 89.9 & 27 & 53 & 06 & 09 & 40.9356 & -15 & 42 & 40.672 & -2 \\
\hline $0607-157$ & & & 4 & 3 & 5 & 4.0 & 12 & BY & 89.9 & $17 \mathrm{~m}$ & 52 & 06 & 09 & 40.9337 & -15 & 42 & 40.690 & -2 \\
\hline $0629-418$ & $\mathrm{Q}$ & 19.3 & 3 & 2 & 5 & 3.2 & 10 & BY & 90.1 & $23 \mathrm{~m}$ & 99 & 06 & 31 & 11.9898 & -41 & 54 & 27.071 & - \\
\hline $0646-306$ & $\ldots$ & $\ldots$ & 3 & 2 & 6 & 3.5 & 22 & $\mathrm{BY}$ & 90.0 & $37 \mathrm{~m}$ & 67 & 06 & 48 & 14.0888 & -30 & 44 & 19.641 & - \\
\hline $0743-006$ & $\mathrm{~L}$ & 17.1 & 3 & 2 & 5 & 3.7 & 9 & BY & 91.1 & $30 \mathrm{~m}$ & 78 & 07 & 45 & 54.0774 & -00 & 44 & 17.746 & - \\
\hline $0743-006$ & & & & 2 & 5 & & 9 & $\mathrm{ZA}$ & 91.0 & $24 \mathrm{~m}$ & 71 & 07 & 45 & 54.0798 & -00 & 44 & 17.742 & - \\
\hline $0743-006$ & & & 4 & 3 & 5 & 4.0 & 9 & BY & 91.1 & $47 \mathrm{~m}$ & 95 & 07 & 45 & 54.0780 & -00 & 44 & 17.718 & - \\
\hline $0743-006$ & & & & 3 & 5 & & 9 & $\mathrm{ZA}$ & 91.0 & $35 \mathrm{~m}$ & 59 & 07 & 45 & 54.0791 & -00 & 44 & 17.714 & - \\
\hline $0818-128$ & $\mathrm{~L}$ & 15 & 3 & 2 & 6 & 3.3 & 5 & BY & 90.2 & $20 \mathrm{~m}$ & 64 & 08 & 20 & 57.4467 & -12 & 58 & 59.173 & - \\
\hline $0906+015$ & $\mathrm{Q}$ & 17.8 & 1 & 2 & 1 & 2.7 & 20 & BY & 91.3 & 12 & 64 & 09 & 09 & 10.0844 & +01 & 21 & 35.379 & -1 \\
\hline $0906+015$ & & & 4 & 2 & 4 & 3.6 & 13 & $\mathrm{BY}$ & 91.3 & $7 \mathrm{~m}$ & 45 & 09 & 09 & 10.0853 & +01 & 21 & 35.434 & - \\
\hline $1004+141$ & $\mathrm{Q}$ & 19 & 1 & 6 & 0 & 3.0 & 31 & $\mathrm{ZA}$ & 87.7 & 19 & 115 & 10 & 07 & 41.4922 & +13 & 56 & 29.482 & - \\
\hline $1039+811$ & $\mathrm{Q}$ & 16.5 & 1 & 2 & 2 & 3.0 & 24 & $\mathrm{ZA}$ & 84.2 & 17 & 162 & 10 & 44 & 22.9495 & +80 & 54 & 39.557 & -2 \\
\hline $1111+149$ & $\mathrm{Q}$ & 18 & 1 & 2 & 1 & 2.9 & 22 & $\mathrm{ZA}$ & 90.3 & 8 & 84 & 11 & 13 & 58.6968 & +14 & 42 & 26.769 & \\
\hline $1656+053$ & $\mathrm{Q}$ & 16.5 & 1 & 2 & 18 & 3.0 & 9 & $\mathrm{ZA}$ & 86.4 & $23 \mathrm{~m}$ & 91 & 16 & 58 & 33.4388 & +05 & 15 & 16.370 & -1 \\
\hline $1800+440$ & $\mathrm{Q}$ & 16.8 & 1 & 3 & 1 & 3.2 & 11 & $\mathrm{ZA}$ & 91.5 & 25 & 70 & 18 & 01 & 32.3140 & +44 & 04 & 22.049 & \\
\hline $2201+315$ & $\mathrm{Q}$ & 15.6 & 2 & 2 & 2 & 2.4 & 13 & $\mathrm{ZA}$ & 87.8 & 48 & 75 & 22 & 03 & 14.9825 & +31 & 45 & 38.293 & \\
\hline
\end{tabular}

Notes to Table 7 .

$\mathrm{t}=$ type of object with $\mathrm{Q}=\mathrm{QSO}, \mathrm{L}=\mathrm{BL} \mathrm{Lac} ; \mathrm{mag}=$ approx. magnitude; run = observing run number (see Table 2 ); $\mathrm{nCCD}=$ numbe $\mathrm{s}=$ short exposure; $\mathrm{FW}=$ mean full width at half maximum of image profiles in pixel; $\sigma_{x y}=$ fit error from a single long exposure in $\mathrm{m}$ $=$ information about secondary reference stars; tel = telescope $(\mathrm{ZA}$ for Hamburg Zone Astrograph, BY for Yellow lens BBAO); yr = m number of reference stars used, with letter $m$ indicating mosaic frames; $\sigma_{C P A}=$ standard error of unit weight of CPA in mas; RA,DC IRS /FK5 system; opt. - radio $=\Delta \alpha \cos \delta, \Delta \delta$ with respect to the radio position of Johnston et al. $(1995) ; \sigma_{Q}=$ estimate of the accuracy o the upcoming Hipparcos catalog and assuming $\sigma s y s_{2}=\sigma s y s_{3}=10$ mas; sep $=$ separation in arcseconds if optically double; flr $=$ flux rat 